\title{
Odd manifolds of small integral simplicial volume
}

\author{
Clara Löh
}

\begin{abstract}
Integral simplicial volume is a homotopy invariant of oriented closed connected manifolds, defined as the minimal weighted number of singular simplices needed to represent the fundamental class with integral coefficients. We show that odd-dimensional spheres are the only manifolds with integral simplicial volume equal to 1 . Consequently, we obtain an elementary proof that, in general, the integral simplicial volume of (triangulated) manifolds is not computable.
\end{abstract}

\section{Introduction}

Simplicial volumes are homotopy invariants for oriented closed connected manifolds, defined as the infimal weighted number of singular simplices needed to represent the fundamental class with respect to the given coefficients. Originally, Gromov introduced the simplicial volume with $\mathbb{R}$-coefficients [10][15][21] in his study of Mostow rigidity. In the context of the connection of simplicial volume with Betti numbers or $L^{2}$-Betti numbers, integral versions of simplicial volume play a central role $[12][16][22]$.

We will consider the most basic integral version: The integral simplicial volume of an oriented closed connected $n$-manifold $M$ is defined as

$$
\|M\|_{\mathbb{Z}}:=\min \left\{|c|_{1} \mid c \in C_{n}(M ; \mathbb{Z}) \text { is a } \mathbb{Z} \text {-fundamental cycle of } M\right\} \in \mathbb{N},
$$

where $|c|_{1}:=\sum_{j=0}^{k}\left|a_{j}\right|$ for any chain $c=\sum_{j=0}^{k} a_{j} \cdot \sigma_{j}$ in $C_{*}(M ; \mathbb{Z})$ (in reduced form). Integral simplicial volume lacks most of the convenient inheritance properties of

This work was supported by the CRC 1085 Higher Invariants (Universität Regensburg, funded by the DFG).

2010 Mathematics Subject Classification: 55N10, 57N65. 
ordinary simplicial volume (such as multiplicativity under finite coverings and gluing formulae), and hence is rather difficult to control.

In the following, we will characterise manifolds with integral simplicial volume equal to 1 , and derive an elementary non-computability statement for integral simplicial volume:

Theorem 1.1. Let $n \in \mathbb{N}_{>0}$ and let $M$ be an oriented closed connected $n$-manifold. Then $\|M\|_{\mathbb{Z}}=1$ if and only if $n$ is odd and $M$ is homotopy equivalent to $S^{n}$.

Remark 1.2. In view of the positive solution of the Poincaré conjecture, the conditions in Theorem 1.1 are also equivalent to $M \cong_{\mathrm{Top}} S^{n}$.

Corollary 1.3. Let $n \in \mathbb{N}_{\geq 5}$ be odd.

1. It is not decidable whether a given triangulated oriented closed connected n-manifold $M$ satisfies $\|M\|_{\mathbb{Z}}=1$.

2. In general, the integral simplicial volume of triangulated oriented closed connected n-manifolds is not computable.

The basic strategy of the proof of Theorem 1.1 is as follows:

- We show that any manifold of integral simplicial volume 1 of dimension $>1$ is simply connected. This step is based on quotients of the standard simplex that model singular cycles consisting of a single simplex (Section 2).

- Similar to the Betti number estimates for integral simplicial volume [19, Example 14.28], we will use Poincaré duality and an explicit formula for the capproduct to prove that any manifold of integral simplicial volume equal to 1 is an integral homology sphere (Lemma 3.1).

- Finally, we apply the Hurewicz and Whitehead theorems to conclude.

More generally, this technique also gives a characterisation for multiples of the fundamental class (Theorem 3.2), which is interesting in the context of integral approximations of simplicial volume (Section 3.3).

We then derive the non-computability statement of Corollary 1.3 from Theorem 1.1 by comparison with the sphere-recognition problem. Alternatively, this result can also be obtained from Weinberger's non-computability result for ordinary simplicial volume of homology spheres, which is based on far less elementary techniques [24, Theorem 2, p. 88].

More concretely, already the case of integral simplicial volume equal to 2 is more involved than Theorem 1.1: For example, in even dimensions, spheres are not the only oriented closed connected manifolds of integral simplicial volume equal to 2 (Proposition 4.6). Moreover, the oriented closed connected 3-manifolds with integral simplicial volume equal to 2 are precisely (up to homotopy/homeomorphism) $S^{1} \times$ $S^{2}, \mathbb{R} P^{3}$, and $L(3,1)$ (Proposition 4.4, Remark 4.5). 


\section{Organisation of this article}

In Section 2, we study the combinatorics of singular cycles that consist of a single singular simplex. Section 3 contains a proof of Theorem 1.1 and a generalisation to multiples of the fundamental class; moreover, we discuss the relation with integral approximations of simplicial volume. In Section 4, we discuss some aspects of the case that the integral simplicial volume equals 2 . Finally, in Section 5, we briefly introduce the relevant setup from computability theory and derive Corollary 1.3.

\section{Model spaces of cycles of a single singular simplex}

Singular cycles that consist of a single singular simplex have a very restricted combinatorial structure. The corresponding model spaces for such cycles are simply connected (Proposition 2.6), but fail to be closed manifolds in general (Proposition 2.7).

\subsection{The model spaces}

A model space associated with a cycle consisting of a single simplex is a standard simplex where the faces are glued according to their cancellation in the singular boundary.

Definition 2.1. (matching, model space) Let $n \in \mathbb{N}$ be odd.

- An $n$-matching is a bijection $\{0,2, \ldots, n-1\} \longrightarrow\{1,3, \ldots, n\}$.

- For $j \in\{0, \ldots, n\}$, we denote the inclusion of the $j$-th face of $\Delta^{n}$ by $i_{j}: \Delta^{n-1} \longrightarrow$ $\Delta^{n}$.

- Let $\pi$ be an $n$-matching. Then the model space associated with $\pi$ is

$$
M_{\pi}:=\Delta^{n} / \sim_{\pi}
$$

where $\sim_{\pi}$ is the equivalence relation generated by

$$
\forall_{j \in\{0,2, \ldots, n-1\}} \forall_{t \in \Delta^{n-1}} \quad i_{j}(t) \sim_{\pi} i_{\pi(j)}(t) .
$$

We denote the canonical projection $\Delta^{n} \longrightarrow M_{\pi}$ by $\sigma_{\pi}$.

Remark 2.2. (model class) Let $n \in \mathbb{N}$ be odd and let $\pi$ be an $n$-matching. Then

$$
\partial_{n} \sigma_{\pi}=\sum_{j=0}^{n}(-1)^{j} \cdot \sigma_{\pi} \circ i_{j}=\sum_{j=0}^{(n-1) / 2}\left(\sigma_{\pi} \circ i_{2 \cdot j}-\sigma_{\pi} \circ i_{\pi(2 \cdot j)}\right)=0
$$


where $\partial_{n}: C_{n}(X ; \mathbb{Z}) \longrightarrow C_{n-1}(X ; \mathbb{Z})$ is the singular boundary operator. Hence, $\sigma_{\pi} \in$ $C_{*}\left(M_{\pi} ; \mathbb{Z}\right)$ is a singular cycle. We write

$$
\alpha_{\pi}:=\left[\sigma_{\pi}\right] \in H_{n}\left(M_{\pi} ; \mathbb{Z}\right) .
$$

Every singular class that is represented by a single singular simplex is a pushforward of one of these model classes:

Lemma 2.3. Let $X$ be a topological space, let $n \in \mathbb{N}_{>0}$, and let $\sigma: \Delta^{n} \longrightarrow X$ be a singular simplex.

1. If $R$ is a ring with unit and $\partial_{n} \sigma=0$ in $C_{*}(X ; R)$, then $n$ is odd.

2. If $\partial_{n} \sigma=0$ in $C_{*}(X ; \mathbb{Z})$, then $n$ is odd and there exist an $n$-matching $\pi$ and a continuous map $f: M_{\pi} \longrightarrow X$ such that

$$
H_{n}(f ; \mathbb{Z})\left(\alpha_{\pi}\right)=[\sigma] \in H_{n}(X ; \mathbb{Z}) .
$$

Proof. Let $R$ be a ring with unit and let $0=\partial_{n} \sigma=\sum_{j=0}^{n}(-1)^{j} \cdot \sigma \circ i_{j}$. Because $\operatorname{map}\left(\Delta^{n-1}, X\right)$ is an $R$-basis of $C_{n-1}(X ; R)$, this sum has an even number of summands; in particular, $n$ is odd.

We now consider the case $R=\mathbb{Z}$. In order for these terms to cancel there has to be a bijection $\pi:\{0,2, \ldots, n-1\} \longrightarrow\{1,3, \ldots, n\}$ such that

$$
\sigma \circ i_{j}=\sigma \circ i_{\pi(j)}
$$

holds for all $j \in\{0,2, \ldots, n-1\}$. Hence, $\sigma$ factors over the projection map $\sigma_{\pi}: \Delta^{n} \longrightarrow$ $\Delta^{n} / \sim_{\pi}=M_{\pi}$, i.e., there is a continuous map $f: M_{\pi} \longrightarrow X$ with $f \circ \sigma_{\pi}=\sigma$. By construction, $H_{n}(f ; \mathbb{Z})\left(\alpha_{\pi}\right)=[\sigma]$.

Remark 2.4. (cellular structure on model spaces) Let $n \in \mathbb{N}$ be odd and let $\pi$ be an $n$-matching. The face lattice of $\Delta^{n}$ induces a CW-structure on the quotient $M_{\pi}=$ $\Delta^{n} / \sim_{\pi}$

Remark 2.5. (trivial extension of matchings) Let $n \in \mathbb{N}$ be odd, let $\pi$ be an $n$-matching, and let $\pi^{\prime}$ be the $(n+2)$-matching with

$$
\left.\pi^{\prime}\right|_{\{0,2, \ldots, n-1\}}:=\pi \text { and } \quad \pi^{\prime}(n+1):=n+2 .
$$

Then $M_{\pi^{\prime}} \cong$ Top $\Sigma^{2} M_{\pi}$, where $\Sigma$ denotes the unreduced suspension. Indeed, this can be seen as follows: Let

$$
\begin{aligned}
\varphi: \Delta^{n} \times[-1,1] & \longrightarrow \Delta^{n+2} \\
(x, t) & \longmapsto \begin{cases}(1-t) \cdot x+t \cdot\left(\frac{1}{4} \cdot e_{n+1}+\frac{3}{4} \cdot e_{n+2}\right) & \text { if } t \geq 0 \\
(1-|t|) \cdot x+|t| \cdot\left(\frac{3}{4} \cdot e_{n+1}+\frac{1}{4} \cdot e_{n+2}\right) & \text { if } t<0 .\end{cases}
\end{aligned}
$$



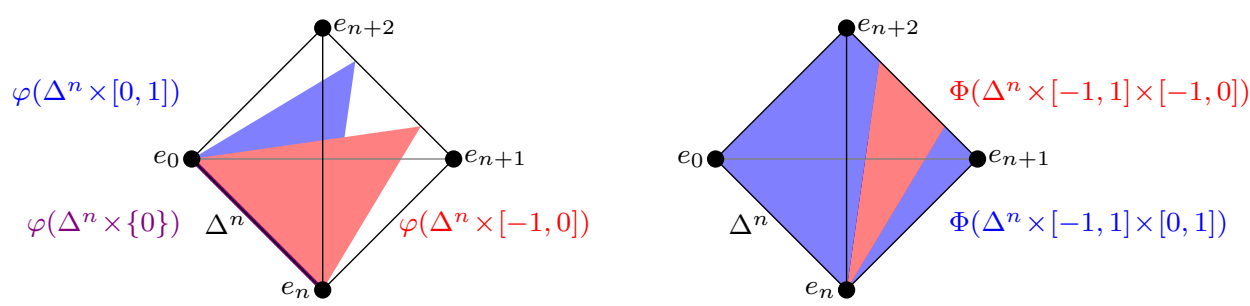

Figure 1. The images of $\varphi$ and $\Phi$ from Remark 2.5. We draw the images in $\Delta^{n+2}$ instead of in $M_{\pi^{\prime}}=\Delta^{n+2} / \sim_{\pi^{\prime}}$.

Then it is not hard to show that the map $\Phi$ given by

$$
\begin{aligned}
& \Delta^{n} \times[-1,1]^{2} \longrightarrow M_{\pi^{\prime}}=\Delta^{n+2} / \sim_{\pi^{\prime}} \\
&(x, t, s) \longmapsto \begin{cases}(1-s) \cdot \varphi(x, t)+s \cdot e_{n+2} & \text { if } t \geq 0, s \geq 0 \\
(1-|s|) \cdot \varphi(x, t)+\frac{|s|}{2} \cdot\left(e_{n+1}+e_{n+2}\right) & \text { if } t \geq 0, s<0 \\
(1-s) \cdot \varphi(x, t)+s \cdot e_{n+1} & \text { if } t<0, s \geq 0 \\
(1-|s|) \cdot \varphi(x, t)+\frac{|s|}{2} \cdot\left(e_{n+1}+e_{n+2}\right) & \text { if } t<0, s<0\end{cases}
\end{aligned}
$$

induces a well-defined homeomorphism $\Sigma^{2} M_{\pi} \cong$ Top $M_{\pi^{\prime}}$ (Figure 1).

There is only one 1-matching, and clearly the corresponding model space is homeomorphic to $S^{1}$. Hence, by induction, for all odd $n \in \mathbb{N}$ the matching $\pi$ given by

$$
0 \longmapsto 1, \quad 2 \longmapsto 3, \quad \ldots, \quad n-1 \longmapsto n
$$

leads to the model space $M_{\pi} \cong$ Top $S^{n}$.

However, not all model spaces are homotopy spheres (Proposition 2.7).

\subsection{Fundamental group of model spaces}

We now show that these model spaces are simply connected:

Proposition 2.6. Let $n \in \mathbb{N}_{\geq 3}$ be odd, and let $\pi$ be an n-matching. Then $M_{\pi}$ is simply connected.

Proof. Clearly, $M_{\pi}$ is connected. We now use the cellular structure from Remark 2.4 to compute the fundamental group of $M_{\pi}$ (with respect to the basepoint given by the 0 -vertex of $\Delta^{n}$ ):

As first step, we describe the 0 -skeleton of $M_{\pi}$ : If $J, K \in\{0, \ldots, n\}$ satisfying $J<$ $K$ are matched through $\pi$, then the $(n-1)$-faces

$$
\begin{aligned}
& {[0, \ldots, J-1, J+1, \ldots, K-1, K, K+1, \ldots, n] \text { and }} \\
& {[0, \ldots, J-1, J, \ldots, K-2, K-1, K+1 \ldots, n]}
\end{aligned}
$$


of $\Delta^{n}$ are glued and so give the same $(n-1)$-cell in $M_{\pi}=\Delta^{n} / \sim_{\pi}$. In particular, the vertices of $\Delta^{n}$ numbered by $J, J+1, \ldots, K$ all project to the same 0 -cell of $M_{\pi}$. Let $\sim$ denote the equivalence relation on $\{0, \ldots, n\}$ generated by

$$
j \sim k \Longleftrightarrow \exists_{J, K \in\{0, \ldots, n\}}(J \leq j \leq k \leq K) \wedge(\pi(J)=K \vee \pi(K)=J) .
$$

Then one can check easily for $j, k \in\{0, \ldots, n\}$ that the $j$-th and the $k$-vertex of $\Delta^{n}$ lead to the same 0 -cell in $M_{\pi}$ if and only if $j \sim k$.

As next step, we describe a spanning tree for the 1-skeleton of $M_{\pi}$ : Using the above description of the 0 -skeleton, it is not hard to see that the 1-cells of $M_{\pi}$ corresponding to the edges

$$
T:=\{[j, j+1] \mid j \in\{0, \ldots, n\} \text { is maximal in its } \sim \text {-equivalence class }\}
$$

of $\Delta^{n}$ is the set of edges of a spanning tree for the 1-skeleton of $M_{\pi}$.

The 2-skeleton of $M_{\pi}$ now gives us the presentation $\pi_{1}\left(M_{\pi}\right) \cong\langle S \mid R\rangle$ of the fundamental group, where

$$
\begin{aligned}
S:= & \left\{s_{[j, k]} \mid j, k \in\{0, \ldots, n\}, j<k\right\} \\
R:= & \left\{s_{e} \mid e \in T\right\} \\
& \cup\left\{s_{[k, \ell]} \cdot s_{[j, k]}=s_{[j, \ell]} \mid j, k, \ell \in\{0, \ldots, n\}, j<k<\ell\right\} \\
& \cup\left\{s_{[j, k]}=s_{\left[j^{\prime}, k^{\prime}\right]} \mid j, k, j^{\prime}, k^{\prime} \in\{0, \ldots, n\}, j<k, j^{\prime}<k^{\prime}, \sigma_{\pi} \circ[j, k]=\sigma_{\pi} \circ\left[j^{\prime}, k^{\prime}\right]\right\} .
\end{aligned}
$$

Using the first and second type of relations, we immediately see that $\pi_{1}\left(M_{\pi}\right)$ is generated by $\left\{s_{[j, j+1]} \mid j \in\{0, \ldots, n-1\},[j, j+1] \notin T\right\}$. So it suffices to show that these elements are trivial in $\pi_{1}\left(M_{\pi}\right)$.

Let $j \in\{0, \ldots, n-1\}$ with $[j, j+1] \notin T$. By construction of $T$, this means that there exist $J, K \in\{0, \ldots, n\}$ with

$$
J \leq j<j+1 \leq K
$$

and the property that $J$ and $K$ are matched through $\pi$. We now consider the following cases:

- Let $K \neq n$. Because $J$ and $K$ are matched through $\pi$, we have

$$
\sigma_{\pi} \circ[j, n]=\sigma_{\pi} \circ[j+1, n],
$$

and so $s_{[j, n]}=s_{[j+1, n]}$ in $\pi_{1}\left(M_{\pi}\right)$. Then, the 2 -face $[j, j+1, n]$ of $\Delta^{n}$ shows that

$$
s_{[j, j+1]}=s_{[j+1, n]}^{-1} \cdot s_{[j, n]}=1
$$

in $\pi_{1}\left(M_{\pi}\right)$.

- The case of $J \neq 0$ can be treated in the same way as the previous case.

- Let $J=0$ and $K=n$. Because $n \geq 3$ there is at least one other matching between some $J^{\prime}, K^{\prime} \in\{0, \ldots, n\}$ with $J^{\prime}<K^{\prime}$ through $\pi$. The same argument as in 
the first case yields $s_{\left[J^{\prime}, J^{\prime}+1\right]}=1$ in $\pi_{1}\left(M_{\pi}\right)$. On the other hand, $\pi(0)=n$ gives

$$
s_{[0,1]}=s_{[1,2]}=\ldots=s_{[n-1, n]}
$$

in $\pi_{1}\left(M_{\pi}\right)$. In particular, $s_{[j, j+1]}=s_{\left[J^{\prime}, J^{\prime}+1\right]}=1$ in $\pi_{1}\left(M_{\pi}\right)$.

Hence, $\pi_{1}\left(M_{\pi}\right)$ is the trivial group.

\subsection{How close are the model spaces to being manifolds?}

These model spaces are (after sufficiently many barycentric subdivisions) $n$-dimensional pseudo-manifolds. However, in general, they do not have the homotopy type of oriented closed connected manifolds:

\section{Proposition 2.7.}

1. Let $n \in\{1,3\}$, and let $\pi$ be an n-matching. Then $M_{\pi}$ is homotopy equivalent to $S^{n}$.

2. For every odd $n \in \mathbb{N}_{\geq 5}$ there exists an n-matching $\pi$ such that the model space $M_{\pi}$ does not have the homotopy type of an oriented closed connected $n$-manifold. In particular, $M_{\pi} \nsucceq S^{n}$.

Proof. Ad 1. The matchings $0 \mapsto 1$ and $0 \mapsto 1,2 \mapsto 3$ lead to $S^{1}$ and $S^{3}$, respectively (Remark 2.5). It remains to consider the matching $\pi$ given by

$$
\begin{aligned}
& 0 \longmapsto 3 \\
& 2 \longmapsto 1 .
\end{aligned}
$$

Recall that any simply connected integral homology sphere is a homotopy sphere by the Hurewicz and Whitehead theorems. By Proposition 2.6 it is thus sufficient to compute $H_{*}\left(M_{\pi} ; \mathbb{Z}\right)$ using the cellular structure from Remark 2.4: A straightforward calculation shows that:

- By Proposition 2.6 , we have $H_{0}\left(M_{\pi} ; \mathbb{Z}\right) \cong \mathbb{Z}$ and $H_{1}\left(M_{\pi} ; \mathbb{Z}\right) \cong 0$.

- The cellular chain complex of $M_{\pi}$ in degree 2 has the 2-cells corresponding to the 2 -faces $[0,1,2]$ and $[0,1,3]$ as a basis; in degree 1 , a basis is given by the 1 -faces $[0,1],[0,3]$, and with respect to these bases, the boundary map is represented by the matrix

$$
\left(\begin{array}{cc}
1 & 2 \\
0 & -1
\end{array}\right)
$$

Thus, there are no cellular cycles in degree 2 and so $H_{2}\left(M_{\pi} ; \mathbb{Z}\right) \cong 0$.

- Because $\pi$ is a matching, the unique 3 -cell of $M_{\pi}$ is a cycle; as $M_{\pi}$ has no cells in dimension at least 4 , it follows that $H_{3}\left(M_{\pi} ; \mathbb{Z}\right) \cong \mathbb{Z}$ and $H_{j}\left(M_{\pi} ; \mathbb{Z}\right) \cong 0$ for all $j \in \mathbb{N}_{\geq 4}$. 
Therefore, $H_{*}\left(M_{\pi} ; \mathbb{Z}\right) \cong H_{*}\left(S^{3} ; \mathbb{Z}\right)$.

Ad 2. We first prove the statement in dimension 5: To this end, we consider the matching $\pi$ given by

$$
\begin{aligned}
& 0 \longmapsto 3 \\
& 2 \longmapsto 5 \\
& 4 \longmapsto 1 .
\end{aligned}
$$

Using the cellular structure from Remark 2.4 one can calculate that

$$
H_{j}\left(M_{\pi} ; \mathbb{Z}\right) \cong\left\{\begin{array}{lll}
\mathbb{Z} & \text { if } j=0 \\
0 & \text { if } j=1 \\
0 & \text { if } j=2 & \\
0 & \text { if } j=3 \\
\mathbb{Z} & \text { if } j=4, & \text { generated by " }[1,2,3,4,5]-[0,1,3,4,5] " \\
\mathbb{Z} & \text { if } j=5, & \text { generated by " }[0, \ldots, 5] " \\
0 & \text { if } j \geq 6 &
\end{array}\right.
$$

for all $j \in \mathbb{N}$. Hence, $H_{*}\left(M_{\pi} ; \mathbb{Z}\right)$ is not compatible with Poincaré duality, and so $M_{\pi}$ cannot have the homotopy type of an oriented closed connected 5-manifold.

Let $k \in \mathbb{N}$. Taking successive trivial extensions of this matching $\pi$ as in Remark 2.5 leads to model spaces homeomorphic to $\Sigma^{2 \cdot k} M_{\pi}$ of dimension $5+2 \cdot k$. Because

$$
H_{j}\left(\Sigma^{2 \cdot k} M_{\pi} ; \mathbb{Z}\right) \cong \begin{cases}0 & \text { if } j \in\{1, \ldots, 3+2 \cdot k\} \\ \mathbb{Z} & \text { if } j \in\{0,4+2 \cdot k, 5+2 \cdot k\}\end{cases}
$$

is not compatible with Poincaré duality, these model spaces do not have the homotopy type of an oriented closed connected $(5+2 \cdot k)$-manifold.

\section{Manifolds of integral simplicial volume equal to 1}

In this section, we will prove Theorem 1.1 and its generalisation to multiples of the fundamental class (Theorem 3.2).

\subsection{Small fundamental class}

We follow the strategy outlined in the introduction. In particular, we will use the model spaces introduced in Section 2 and the following refinement of the classical Betti number bound [19, Example 14.28]: 
Lemma 3.1. Let $n \in \mathbb{N}$ be odd and let $M$ be an oriented closed connected $n$-manifold.

1. Let $R$ be a ring with unit, let $m \in \mathbb{Z}$ be invertible in $R$, and suppose that $\left\|m \cdot[M]_{\mathbb{Z}}\right\|_{1, \mathbb{Z}}=1$. Then for all $k \in\{1, \ldots, n-1\}$ we have

$$
H_{k}(M ; R) \cong 0
$$

2. In particular: If $\|M\|_{\mathbb{Z}}=1$, then for all $k \in\{1, \ldots, n-1\}$ we have

$$
H_{k}(M ; \mathbb{Z}) \cong 0
$$

If $X$ is a space and $\alpha \in H_{*}(X ; \mathbb{Z})$, then

$$
\|\alpha\|_{1, \mathbb{Z}}:=\min \left\{|c|_{1} \mid c \in C_{*}(X ; \mathbb{Z}) \text { is a cycle representing } \alpha\right\} .
$$

For example, $\|M\|_{\mathbb{Z}}=\left\|[M]_{\mathbb{Z}}\right\|_{1, \mathbb{Z}}$ holds for all oriented closed connected manifolds $M$.

Proof. It suffices to prove the first part. Because of $\left\|m \cdot[M]_{\mathbb{Z}}\right\|_{1, \mathbb{Z}}=1$ there is a singular simplex $\sigma: \Delta^{n} \longrightarrow M$ that is a singular cycle and that satisfies $[\sigma]= \pm m$. $[M]_{\mathbb{Z}} \in H_{n}(M ; \mathbb{Z})$; without loss of generality, we may assume $[\sigma]=m \cdot[M]_{\mathbb{Z}}$. Because $m$ is a unit in $R$, the Poincaré duality homomorphism

$$
\begin{aligned}
& \varphi_{k}:=\cdot \cap m \cdot[M]_{R}: H^{n-k}(M ; R) \longrightarrow H_{k}(M ; R) \\
& {[f] \longmapsto(-1)^{(n-k) \cdot k} \cdot\left[f\left({ }_{n-k}\lfloor\sigma) \cdot \sigma\right\rfloor_{k}\right] }
\end{aligned}
$$

with $R$-coefficients is an isomorphism for all $k \in\{0, \ldots, n\}$. Hence, it is enough to show that $\varphi_{k}$ is the zero map for all $k \in\{1, \ldots, n-1\}$ : If $k$ is even, then the $k$-simplex $\sigma\rfloor_{k}$ cannot be cycle (Lemma 2.3), and so the fact that $\varphi_{k}$ as described above is well-defined shows that $\varphi_{k}=0 .\left(^{1}\right)$

Now let $k \in\{1, \ldots, n-1\}$ be odd and let $\pi$ be an $n$-matching for $\sigma$ as in Lemma 2.3. We then define $I:=\{j \in\{0,2, \ldots, k+1\} \mid \pi(j) \leq k\}$ and $\bar{I}:=\{0, \ldots, k+1\} \backslash(I \cup$ $\pi(I))$. Moreover, for a sequence $\left(j_{0}, \ldots, j_{r}\right)$ in $\{0, \ldots, n\}$ we write

$$
\begin{aligned}
{\left[j_{0}, \ldots, j_{r}\right]_{\sigma}: \Delta^{r} } & \longrightarrow M \\
\left(t_{0}, \ldots, t_{r}\right) & \longmapsto \sigma\left(\sum_{s=0}^{r} t_{s} \cdot e_{j_{s}}\right)
\end{aligned}
$$

for the corresponding subsimplex of $\sigma$, where, $e_{0}, \ldots, e_{n}$ denote the standard unit vectors of $\mathbb{R}^{n+1} \supset \Delta^{n}$. For example, $\left.\sigma\right\rfloor_{k}=[0, \ldots, k]_{\sigma}$. With this notation and the relation $\sigma \circ i_{j}=\sigma \circ i_{\pi(j)}$ for all $j \in\{0,2, \ldots, n-1\}$, we obtain

$\left({ }^{1}\right)$ Alternatively, one can use the arguments for the odd case to show that ${ }_{n-k}\lfloor\sigma$ is a boundary, whence every cocycle vanishes on ${ }_{n-k}\lfloor\sigma$. 


$$
\begin{aligned}
\partial_{k+1} & {[0, \ldots, k+1]_{\sigma} } \\
& =\sum_{j=0}^{k+1}(-1)^{j} \cdot[0, \ldots, \hat{j}, \ldots, k+1]_{\sigma} \\
& =\sum_{j \in I}[0, \ldots, \widehat{\pi(j)}, \ldots, k+1]_{\sigma}-\sum_{j \in \pi(I)}[0, \ldots, \hat{j}, \ldots k+1]_{\sigma}+\sum_{j \in \bar{I}}(-1)^{j} \cdot[0, \ldots, k]_{\sigma} \\
& \left.=0+\sum_{j \in \bar{I}}(-1)^{j} \cdot \sigma\right\rfloor_{k} .
\end{aligned}
$$

Because $k$ is odd, also $|\bar{I}|$ is odd; moreover, $\pi$ matches even with odd indices, so the number of even elements and the number of odd elements in $\bar{I}$ differ by 1 . Therefore,

$$
\left.\partial_{k+1}[0, \ldots, k+1]_{\sigma}= \pm \sigma\right\rfloor_{k} .
$$

Thus, $\left.[\sigma\rfloor_{k}\right]=0$ in $H_{k}(M ; R)$, which implies $\varphi_{k}=0$.

Proof of Theorem 1.1. Let $n \in \mathbb{N}$ be odd, and let $M$ be an oriented closed connected $n$-manifold with $M \simeq S^{n}$. Then $\|M\|_{\mathbb{Z}}=\left\|S^{n}\right\|_{\mathbb{Z}}$. Moreover, $\left\|S^{n}\right\|_{\mathbb{Z}}=1$ for odd $n$, as is witnessed by the cycle $\Delta^{n} \longrightarrow \Delta^{n} / \partial \Delta^{n} \cong$ Top $S^{n}$ given by the canonical projection.

Conversely, let $n \in \mathbb{N}_{>0}$ and $M$ be an oriented closed connected $n$-manifold that satisfies $\|M\|_{\mathbb{Z}}=1$. Then $n$ is odd by Lemma 2.3. Because $S^{1}$ is the only oriented closed connected 1-manifold we may assume $n \geq 3$. We follow the strategy outlined in the introduction:

The manifold $M$ is simply connected: In view of Lemma 2.3 there is an $n$-matching $\pi$ and a continuous map $f: M_{\pi} \longrightarrow$ such that

$$
H_{n}(f ; \mathbb{Z})\left(\alpha_{\pi}\right)=[M]_{\mathbb{Z}} \in H_{n}(M ; \mathbb{Z})
$$

Because $M_{\pi}$ is simply connected, $f$ lifts to a map $\tilde{f}: M_{\pi} \longrightarrow \widetilde{M}$ to the universal covering $p: \widetilde{M} \longrightarrow M$ of $M$. Thus,

$$
H_{n}(p ; \mathbb{Z}) \circ H_{n}(\tilde{f} ; \mathbb{Z})\left(\alpha_{\pi}\right)=H_{n}(f ; \mathbb{Z})\left(\alpha_{\pi}\right)=[M]_{\mathbb{Z}} \neq 0,
$$

and so $H_{n}(\widetilde{M} ; \mathbb{Z}) \neq 0$. In particular, $\widetilde{M}$ is compact and $p$ is a finite covering. Transfer shows that im $H_{n}(p ; \mathbb{Z})=D \cdot \mathbb{Z} \cdot[M]_{\mathbb{Z}}$, where $D$ is the number of sheets of $\pi$. Therefore, $D=1$, and so $M \cong$ Top $\widetilde{M}$ is simply connected.

We have $H_{*}(M ; \mathbb{Z}) \cong H_{*}\left(S^{n} ; \mathbb{Z}\right)$ by Lemma 3.1 .

Hence, $M \simeq S^{n}$ : Any simply connected integral homology $n$-sphere is homotopy equivalent to $S^{n}$ by the Hurewicz and Whitehead theorems. 


\subsection{Small multiples of the fundamental class}

More generally, this technique also gives the following characterisation for multiples of the fundamental class:

Theorem 3.2. (multiples of the fundamental class of small integral $\ell^{1}$-norm) Let $n \in \mathbb{N}$ be odd and let $M$ be an oriented closed connected $n$-manifold. Then the following are equivalent:

1. There exists $m \in \mathbb{Z} \backslash\{0\}$ with $\left\|m \cdot[M]_{\mathbb{Z}}\right\|_{1, \mathbb{Z}}=1$.

2. The manifold $M$ is dominated by $S^{n}$, i.e., there is a map $S^{n} \longrightarrow M$ of nonzero degree.

3. There is a subsequence of $\left(\left\|m \cdot[M]_{\mathbb{Z}}\right\|_{1, \mathbb{Z}}\right)_{m \in \mathbb{N}}$ that is bounded by 1.

Proof. The implication " $3 \Longrightarrow 1$ " is clear. For the implication " $2 \Longrightarrow 3$ " we use self-maps $S^{n} \longrightarrow S^{n}$ of arbitrarily high degree, the fact that $\left\|S^{n}\right\|_{\mathbb{Z}}=1$ and that

$$
\left\|H_{*}(f ; \mathbb{Z})(\alpha)\right\|_{1, \mathbb{Z}} \leq\|\alpha\|_{1, \mathbb{Z}}
$$

holds for all continuous maps $f: X \longrightarrow Y$ and all $\alpha \in H_{*}(X ; \mathbb{Z})$.

We will now prove " $1 \Longrightarrow 2$ ": Without loss of generality we may assume that $n>1$. Let $m \in \mathbb{Z} \backslash\{0\}$ with $\left\|m \cdot[M]_{\mathbb{Z}}\right\|_{1, \mathbb{Z}}=1$. We will now proceed as in the proof of Theorem 1.1:

- Reduction to the simply connected case: Because $\left\|m \cdot[M]_{\mathbb{Z}}\right\|_{1, \mathbb{Z}}=1$, Lemma 2.3 implies that there is an $n$-matching $\pi$ and a continuous map $f: M_{\pi} \longrightarrow M$ satisfying

$$
H_{n}(f ; \mathbb{Z})\left(\alpha_{\pi}\right)=m \cdot[M]_{\mathbb{Z}}
$$

The model space $M_{\pi}$ is simply connected (Proposition 2.6); hence, $f$ lifts to a map $\tilde{f}: M_{\pi} \longrightarrow \widetilde{M}$ to the universal covering $\widetilde{M}$ of $M$. Because $m \cdot[M]_{\mathbb{Z}} \neq 0$ it follows that $H_{n}(\widetilde{M} ; \mathbb{Z}) \nsucceq 0$, and so $\widetilde{M}$ is compact and $H_{n}(\tilde{f} ; \mathbb{Z})\left(\alpha_{\pi}\right)$ is a non-zero multiple of $[\widetilde{M}]_{\mathbb{Z}}$ with integral $\ell^{1}$-norm equal to 1 .

- We have $H_{*}(\widetilde{M} ; \mathbb{Q}) \cong H_{*}\left(S^{n} ; \mathbb{Q}\right)$ by Lemma 3.1 and the first step.

- Existence of a non-zero degree map $g: S^{n} \longrightarrow \widetilde{M}$ : Combining the previous steps with the rational Hurewicz theorem [6, Theorem 8.6] implies that the rational Hurewicz homomorphism

$$
\begin{aligned}
& h_{n}^{\widetilde{M}}: \pi_{n}(\widetilde{M}) \otimes_{\mathbb{Z}} \mathbb{Q} \longrightarrow H_{n}(\widetilde{M} ; \mathbb{Z}) \otimes_{\mathbb{Z}} \mathbb{Q} \cong H_{n}(\widetilde{M} ; \mathbb{Q}) \\
& {[g]_{*} \longmapsto H_{n}(g ; \mathbb{Z})\left(\left[S^{n}\right]_{\mathbb{Z}}\right) \otimes 1 }
\end{aligned}
$$

is surjective, and any (pointed) continuous map $g: S^{n} \longrightarrow \widetilde{M}$ that is mapped via $h_{n}^{\widetilde{M}}$ to an element of $H_{n}(\widetilde{M} ; \mathbb{Q}) \backslash\{0\}$ has non-zero degree.

Composing $g: S^{n} \longrightarrow \widetilde{M}$ with the universal covering map $\widetilde{M} \longrightarrow M$ hence shows that $M$ is dominated by $S^{n}$. 


\subsection{Integral approximations of simplicial volume}

Determining the exact relation between ordinary simplicial volume and $L^{2}$-Betti numbers of oriented closed connected aspherical manifolds is a long-standing open problem [11, p. 232]. Estimates between Betti numbers and simplicial volumes can be obtained in the presence of some integrality of coefficients [12][22], based on Poincaré duality and a counting argument as in Lemma 3.1. Hence, we are led to the question in which sense ordinary simplicial volume can be approximated by integral versions of simplicial volume and how these approximations relate to Betti numbers and $L^{2}$-Betti numbers.

On the one hand, one can use the action of the fundamental group to introduce more flexibility in the coefficients. This leads to stable integral simplicial volume (based on the integral simplicial volumes of all finite coverings) and integral foliated simplicial volume (based on probability spaces with an action of the fundamental group) [7][16]. In contrast to $L^{2}$-Betti numbers that can be computed by the corresponding integral approximations [18][9], stable integral simplicial volume and integral foliated simplicial volume of oriented closed connected hyperbolic manifolds of dimension at least 4 do not coincide with ordinary simplicial volume [7][8].

On the other hand, ordinary simplicial volume of an oriented closed connected manifold $M$ coincides with [17, Remark 5.4]

$$
\inf _{d \in \mathbb{N}_{>0}} \frac{1}{d} \cdot\left\|d \cdot[M]_{\mathbb{Z}}\right\|_{1, \mathbb{Z}}
$$

Therefore, one can use the sequence $\Sigma(M):=\left(\left\|d \cdot[M]_{\mathbb{Z}}\right\|_{1, \mathbb{Z}}\right)_{d \in \mathbb{N}}$ to introduce refined versions of vanishing of simplicial volume. The strongest such vanishing occurs in case the sequence $\Sigma(M)$ contains a subsequence bounded by 1 . This is exactly the situation of Theorem 3.2, which gives a complete geometric characterisation of such manifolds. More generally, the property that $\Sigma(M)$ contains a bounded subsequence is related to weak flexibility of homology classes [17, Theorem 5.1, Remark 5.4][5]. However, it is not clear how $\Sigma(M)$ is related to $L^{2}$-Betti numbers in the aspherical case.

\section{Manifolds of integral simplicial volume equal to 2}

In the previous section, we gave a complete classification of manifolds with integral simplicial volume equal to 1 . The natural next step is to look at manifolds with integral simplicial volume equal to 2 .

Question 4.1 . Which oriented closed connected manifolds $M$ satisfy $\|M\|_{\mathbb{Z}}=2$ ? 

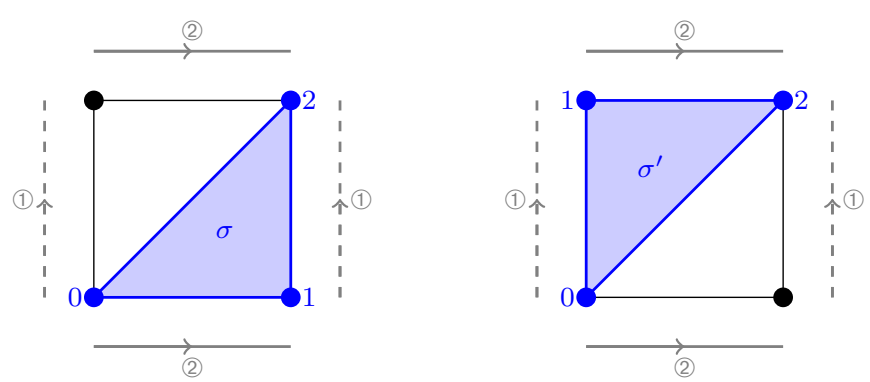

Figure 2. Fundamental cycle $\sigma-\sigma^{\prime}$ of the torus; the edges of the square are glued as indicated

However, this problem seems to be much harder than the case of integral simplicial volume equal to 1 . In the following, we will give some partial answers.

We begin with a small, but helpful, observation, generalising the parity aspect of Lemma 2.3:

Lemma 4.2. (parity of cycles) Let $M$ be an oriented closed connected manifold of even dimension. Then $\|M\|_{\mathbb{Z}}$ is even.

Proof. Let $n:=\operatorname{dim} M$ and let $c=\sum_{j=1}^{k} a_{j} \cdot \sigma_{j} \in C_{n}(M ; \mathbb{Z})$ be an integral fundamental cycle. Because $\Delta^{n}$ has an odd number of $(n-1)$-faces and because the singular $n$-simplices of $M$ form a $\mathbb{Z}$-basis of $C_{n}(M ; \mathbb{Z})$, the definition of the boundary operator yields that $\sum_{j=1}^{k} a_{j}$ is even. Then also $|c|_{1}=\sum_{j=1}^{k}\left|a_{j}\right|$ is even and thus $\|M\|_{\mathbb{Z}}$ is even.

\subsection{Dimension 2}

We start with the simple and well-known case of dimension 2:

Proposition 4.3. (integral simplicial volume of surfaces)

1. We have $\left\|S^{2}\right\|_{\mathbb{Z}}=2$.

2. If $S$ is an oriented closed connected surface of genus $g \in \mathbb{N}_{\geq 1}$, then $\|S\|_{\mathbb{Z}}=$ $4 \cdot g-2$.

Proof. In even dimension, there is no cycle consisting of a single simplex (Lemma 2.3). Thus, oriented closed connected surfaces $S$ satisfy $\|S\|_{\mathbb{Z}} \geq 2$. Clearly, $\left\|S^{2}\right\|_{\mathbb{Z}}=2$ and $\left\|S^{1} \times S^{1}\right\|_{\mathbb{Z}}=2$; the latter can be seen from the fundamental cycle depicted in Figure 2.

Let $S$ be an oriented closed connected surface of genus $g \in \mathbb{N}_{>1}$. Then there is an explicit triangulation of the regular $4 \cdot g$-gon that shows that $\|S\|_{\mathbb{Z}} \leq 4 \cdot g-2$ 
[10][2]. On the other hand, we have

$$
4 \cdot g-4=\|S\| \leq\|S\|_{\mathbb{Z}} \cdot
$$

No fundamental cycle on $S$ realises the optimal value $\|S\|=4 \cdot g-4$ because no straight singular simplex on $\mathbb{H}^{2}$ has maximal volume [13]. Therefore, $\|S\|_{\mathbb{Z}}>4 \cdot g-4$. Because $\|S\|_{\mathbb{Z}}$ is even (Lemma 4.2 ), we obtain the desired estimate $\|S\|_{\mathbb{Z}} \geq 4 \cdot g-2$.

\subsection{Dimension 3}

As a next step, we consider 3-manifolds.

Proposition 4.4. The following oriented closed connected 3-manifolds $M$ satisfy $\|M\|_{\mathbb{Z}}=2$ :

$$
S^{1} \times S^{2}, \quad \mathbb{R} P^{3}, \quad L(3,1) .
$$

Proof. In view of Theorem 1.1, all of these manifolds have integral simplicial volume at least 2 .

Ad $S^{1} \times S^{2}$ : By Proposition 4.6 below, $\left\|S^{1} \times S^{2}\right\|_{\mathbb{Z}}=2$.

Ad $\mathbb{R} P^{3}$ : We describe an integral fundamental cycle for $\mathbb{R} P^{3}$ that consists of two singular simplices: We view $\mathbb{R} P^{3}$ as quotient of the 3 -ball $D^{3}$ modulo the antipodal action on $\partial D^{3}=S^{2}$. We consider the singular 3 -simplices $\sigma, \sigma^{\prime}$ on $\mathbb{R} P^{3}$ as depicted in Figure 3. Then

$$
\partial_{3}\left(\sigma+\sigma^{\prime}\right)=0
$$

and looking at the local degree shows that $\sigma+\sigma^{\prime}$ represents $\left[\mathbb{R} P^{3}\right]_{\mathbb{Z}}$. In fact, we even have that the corresponding model space (in the sense of Remark 4.5) is homeomorphic to $\mathbb{R} P^{3}$.

Ad $L(3,1)$ : The construction of a fundamental cycle of $L(3,1)$ consisting of two singular simplices is a little bit more delicate: We view the lens space $L(3,1)$ as quotient of the 3 -ball $D^{3}$, where points on the southern hemisphere of $\partial D^{3}=S^{2}$ are identified with points on the northern hemisphere after reflection at the equator and rotating around the North-South axis by $2 \cdot \pi / 3$. We consider the two singular 3 -simplices $\sigma, \sigma^{\prime \prime}$ on $L(3,1)$ as depicted in Figure 4.

By construction, $\partial_{3}\left(\sigma+\sigma^{\prime \prime}\right)=0$ and looking at the local degree (inside the ball) shows that $\sigma+\sigma^{\prime \prime}$ represents $2 \cdot[L(3,1)]_{\mathbb{Z}}$. We now manipulate the local degree of $\sigma^{\prime \prime}$ in order to obtain a fundamental cycle of $L(3,1)$ : We may construct $\sigma^{\prime \prime}$ in such a way that $\sigma^{\prime \prime}$ on the middle slice

$$
S:=\left\{\left(t_{0}, t_{1}, t_{2}, t_{3}\right) \in \mathbb{R}^{4} \mid t_{0}+\ldots+t_{3}=1, t_{0}=t_{3}\right\}
$$



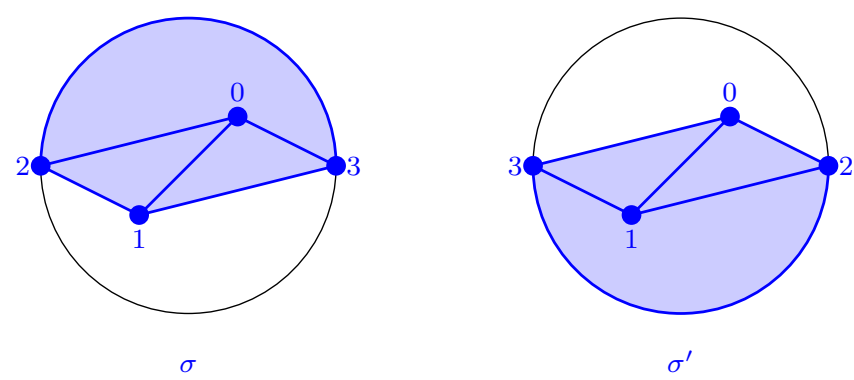

Figure 3. A fundamental cycle of $\mathbb{R} P^{3}$ consisting of two singular simplices: The simplex $\sigma$ fills the northern part of the ball with the depicted combinatorial structure; here, the face $\sigma \circ i_{0}$ is the front half of the northern hemisphere, and $\sigma \circ i_{1}$ is the back half of the northern hemisphere. Similarly, the simplex $\sigma^{\prime}$ fills the southern part of the ball.

of $\Delta^{3}$ is the obvious parametrisation of the equatorial "disk" in $D^{3}$. Let $P$ be the $\Delta^{2}$-shaped pillow obtained from $\Delta^{2} \times[0,1]$ by collapsing the vertical intervals in $\partial \Delta^{2} \times[0,1]$. One can construct a continuous map $\tau: P \longrightarrow L(3,1)$ that coincides with $\left.\sigma^{\prime \prime}\right|_{S}$ on the top and the bottom of $P$ and that has local degree -3 , as follows: The universal covering of $L(3,1)$ is $S^{3}$ (through a 3 -sheeted covering). We can construct a continuous map $\tilde{\tau}: P \longrightarrow S^{3}$ of local degree -1 that, on the top and bottom of the pillow $P$, covers $\left.\sigma^{\prime \prime}\right|_{S}$ (this can be done by looking at the cellular structure on $S^{3}$ induced by the standard cellular structure on $\left.L(3,1)\right)$. Hence, $\tilde{\tau}$ pushes down to a map $\tau: P \longrightarrow L(3,1)$ with the desired properties. We then glue $\tau$ into $\sigma^{\prime \prime}$ at $S$; because gluing $P$ into $\Delta^{3}$ at $S$ yields a space that is homeomorphic to $\Delta^{3}$ (while preserving the boundary $\left.\partial \Delta^{3}\right)$, we thus obtain a singular simplex $\sigma^{\prime}: \Delta^{3} \longrightarrow L(3,1)$ of local degree $1-3=-2$ with $\partial_{3} \sigma^{\prime}=\partial_{3} \sigma^{\prime \prime}$.

Then $\partial_{3}\left(-\sigma-\sigma^{\prime}\right)=0$ and looking at the local degree shows that the cycle $-\sigma-$ $\sigma^{\prime}$ represents $[L(3,1)]_{\mathbb{Z}}$.

The lens space $L(3,1)$ admits an obvious deomposition into two tetrahedra; however, it is not hard to see that there is no affine parametrisation of these tetrahedra that yields an integral fundamental cycle of $L(3,1)$.

Remark 4.5. If $M$ is an oriented closed connected 3-manifold that is not homotopy equivalent/homeomorphic to $S^{1} \times S^{2}, \mathbb{R} P^{3}$ or $L(3,1)$, then we have $\|M\|_{\mathbb{Z}} \neq 2$ : The model space construction of Section 2 can be extended to the case of singular cycles that consist of more than one simplex. If $M$ has a fundamental cycle $c$ consisting of two singular simplices $\sigma, \sigma^{\prime}: \Delta^{3} \longrightarrow M$, then (depending on the signs of the coefficients) there are two types of matchings to be considered:

- If $c=\sigma+\sigma^{\prime}$ (or $\left.c=-\sigma-\sigma^{\prime}\right)$, then one obtains a bijection

$$
\left\{0,2,0^{\prime}, 2^{\prime}\right\} \longrightarrow\left\{1,3,1^{\prime}, 3^{\prime}\right\}
$$



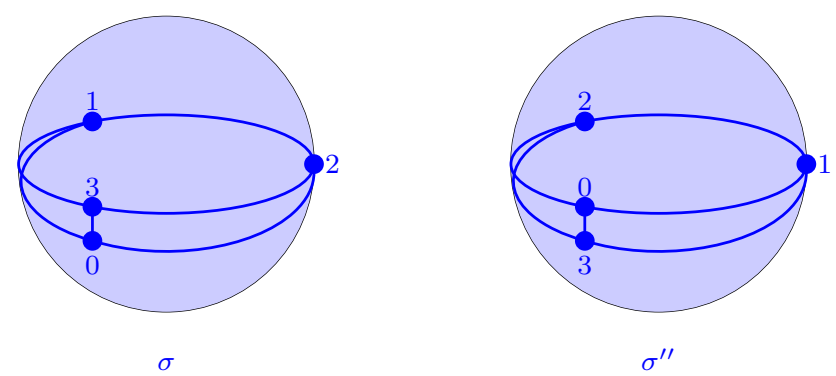

Figure 4. A fundamental cycle of $L(3,1)$ consisting of two singular simplices: The simplex $\sigma$ fills the ball, where $\sigma \circ i_{0}$ covers the northern hemisphere, $\sigma \circ i_{3}$ covers the southern hemisphere and the faces $\sigma \circ i_{1}, \sigma \circ i_{2}$ lie on the equator. The simplex $\sigma^{\prime \prime}$ is constructed in a similar way; here, $\sigma^{\prime \prime} \circ i_{0}$ covers the southern hemisphere and $\sigma^{\prime \prime} \circ i_{3}$ covers the northern hemisphere. The simplex $\sigma^{\prime}$ is then obtained from $\sigma^{\prime \prime}$ by inserting a pillow of local degree -3 .

where the sets $\{0, \ldots, n\}$ and $\left\{0^{\prime}, \ldots, n^{\prime}\right\}$ correspond to the faces of $\sigma$ and $\sigma^{\prime}$ respectively.

- If $c=\sigma-\sigma^{\prime}$, then one obtains a bijection

$$
\left\{0,2,1^{\prime}, 3^{\prime}\right\} \longrightarrow\left\{1,3,0^{\prime}, 2^{\prime}\right\}
$$

Similar to the proof of Proposition 2.6, a lengthy calculation shows that the (connected components of the) corresponding model spaces constructed out of two copies of $\Delta^{3}$ whose facets are glued according to these matchings have cyclic fundamental group. Moreover, we obtain (e.g., by computer calculations) the integral homology groups of these model spaces in Table 1 and Table 2.

Hence, all these model spaces have first integral homology group isomorphic to

$$
0, \quad \mathbb{Z} / 2, \quad \mathbb{Z} / 3, \quad \text { or } \mathbb{Z} \text {. }
$$

Because these model spaces have cyclic (in particular, Abelian) fundamental group, these are also the only possible isomorphism types of fundamental groups of such model spaces.

Let $\pi$ be the matching corresponding to the considered fundamental cycle $c$ of $M$, let $N_{\pi}=\Delta^{3} \sqcup \Delta^{3} / \sim$ be the associated model space, let $\beta_{\pi} \in H_{3}\left(N_{\pi} ; \mathbb{Z}\right)$ be the model class and let $f: N_{\pi} \longrightarrow M$ be the continuous map modelling $c$. Then $H_{3}(f ; \mathbb{Z})\left(\beta_{\pi}\right)=[M]_{\mathbb{Z}}$, and

$$
\begin{array}{r}
H_{k}\left(N_{\pi} ; \mathbb{Z}\right) \stackrel{H_{k}(f ; \mathbb{Z})}{\longrightarrow} H_{k}(M ; \mathbb{Z}) \\
\cdot \cap \beta_{\pi} \uparrow \underset{\uparrow}{\cong} \cdot \cap[M]_{\mathbb{Z}} \\
H^{3-k}\left(N_{\pi} ; \mathbb{Z}\right)_{H^{\overleftarrow{3-k}(f ; \mathbb{Z})}}^{\overleftarrow{ }} H^{3-k}(M ; \mathbb{Z})
\end{array}
$$


Table 1. Integral homology of model spaces for cycles consisting of two singular 3-simplices with the same sign.

\begin{tabular}{|c|c|c|c|c|c|c|c|}
\hline \multirow{2}{*}{\multicolumn{4}{|c|}{ Matching }} & \multicolumn{4}{|c|}{$H_{*}(\cdot ; \mathbb{Z})$ in degree } \\
\hline & & & & 0 & 1 & 2 & 3 \\
\hline$(0,1)$ & $(2,3)$ & $\left(0^{\prime}, 1^{\prime}\right)$ & $\left(2^{\prime}, 3^{\prime}\right)$ & $\mathbb{Z}^{2}$ & 0 & 0 & $\mathbb{Z}^{2}$ \\
\hline$(0,3)$ & $(2,1)$ & $\left(0^{\prime}, 1^{\prime}\right)$ & $\left(2^{\prime}, 3^{\prime}\right)$ & $\mathbb{Z}^{2}$ & 0 & 0 & $\mathbb{Z}^{2}$ \\
\hline$\left(0,1^{\prime}\right)$ & $(2,3)$ & $\left(0^{\prime}, 1\right)$ & $\left(2^{\prime}, 3^{\prime}\right)$ & $\mathbb{Z}$ & 0 & 0 & $\mathbb{Z}$ \\
\hline$(0,3)$ & $\left(2,1^{\prime}\right)$ & $\left(0^{\prime}, 1\right)$ & $\left(2^{\prime}, 3^{\prime}\right)$ & $\mathbb{Z}$ & 0 & $\mathbb{Z}$ & $\mathbb{Z}$ \\
\hline$\left(0,1^{\prime}\right)$ & $(2,1)$ & $\left(0^{\prime}, 3\right)$ & $\left(2^{\prime}, 3^{\prime}\right)$ & $\mathbb{Z}$ & 0 & 0 & $\mathbb{Z}$ \\
\hline$(0,1)$ & $\left(2,1^{\prime}\right)$ & $\left(0^{\prime}, 3\right)$ & $\left(2^{\prime}, 3^{\prime}\right)$ & $\mathbb{Z}$ & 0 & 0 & $\mathbb{Z}$ \\
\hline$\left(0,3^{\prime}\right)$ & $\left(2,1^{\prime}\right)$ & $\left(0^{\prime}, 3\right)$ & $\left(2^{\prime}, 1\right)$ & $\mathbb{Z}$ & 0 & $\mathbb{Z}$ & $\mathbb{Z}$ \\
\hline$\left(0,1^{\prime}\right)$ & $\left(2,3^{\prime}\right)$ & $\left(0^{\prime}, 3\right)$ & $\left(2^{\prime}, 1\right)$ & $\mathbb{Z}$ & 0 & $\mathbb{Z}^{2}$ & $\mathbb{Z}$ \\
\hline$\left(0,1^{\prime}\right)$ & $(2,3)$ & $\left(0^{\prime}, 3^{\prime}\right)$ & $\left(2^{\prime}, 1\right)$ & $\mathbb{Z}$ & 0 & $\mathbb{Z}$ & $\mathbb{Z}$ \\
\hline$\left(0,3^{\prime}\right)$ & $(2,3)$ & $\left(0^{\prime}, 1^{\prime}\right)$ & $\left(2^{\prime}, 1\right)$ & $\mathbb{Z}$ & 0 & 0 & $\mathbb{Z}$ \\
\hline$(0,3)$ & $\left(2,3^{\prime}\right)$ & $\left(0^{\prime}, 1^{\prime}\right)$ & $\left(2^{\prime}, 1\right)$ & $\mathbb{Z}$ & 0 & $\mathbb{Z}$ & $\mathbb{Z}$ \\
\hline$(0,3)$ & $\left(2,1^{\prime}\right)$ & $\left(0^{\prime}, 3^{\prime}\right)$ & $\left(2^{\prime}, 1\right)$ & $\mathbb{Z}$ & $\mathbb{Z} / 3$ & 0 & $\mathbb{Z}$ \\
\hline$\left(0,3^{\prime}\right)$ & $(2,1)$ & $\left(0^{\prime}, 3\right)$ & $\left(2^{\prime}, 1^{\prime}\right)$ & $\mathbb{Z}$ & 0 & 0 & $\mathbb{Z}$ \\
\hline$(0,1)$ & $\left(2,3^{\prime}\right)$ & $\left(0^{\prime}, 3\right)$ & $\left(2^{\prime}, 1^{\prime}\right)$ & $\mathbb{Z}$ & 0 & 0 & $\mathbb{Z}$ \\
\hline$(0,1)$ & $(2,3)$ & $\left(0^{\prime}, 3^{\prime}\right)$ & $\left(2^{\prime}, 1^{\prime}\right)$ & $\mathbb{Z}^{2}$ & 0 & 0 & $\mathbb{Z}^{2}$ \\
\hline$\left(0,3^{\prime}\right)$ & $(2,3)$ & $\left(0^{\prime}, 1\right)$ & $\left(2^{\prime}, 1^{\prime}\right)$ & $\mathbb{Z}$ & 0 & 0 & $\mathbb{Z}$ \\
\hline$(0,3)$ & $\left(2,3^{\prime}\right)$ & $\left(0^{\prime}, 1\right)$ & $\left(2^{\prime}, 1^{\prime}\right)$ & $\mathbb{Z}$ & $\mathbb{Z} / 2$ & 0 & $\mathbb{Z}$ \\
\hline$(0,3)$ & $(2,1)$ & $\left(0^{\prime}, 3^{\prime}\right)$ & $\left(2^{\prime}, 1^{\prime}\right)$ & $\mathbb{Z}^{2}$ & 0 & 0 & $\mathbb{Z}^{2}$ \\
\hline$\left(0,3^{\prime}\right)$ & $(2,1)$ & $\left(0^{\prime}, 1^{\prime}\right)$ & $\left(2^{\prime}, 3\right)$ & $\mathbb{Z}$ & 0 & 0 & $\mathbb{Z}$ \\
\hline$(0,1)$ & $\left(2,3^{\prime}\right)$ & $\left(0^{\prime}, 1^{\prime}\right)$ & $\left(2^{\prime}, 3\right)$ & $\mathbb{Z}$ & 0 & 0 & $\mathbb{Z}$ \\
\hline$(0,1)$ & $\left(2,1^{\prime}\right)$ & $\left(0^{\prime}, 3^{\prime}\right)$ & $\left(2^{\prime}, 3\right)$ & $\mathbb{Z}$ & 0 & $\mathbb{Z}$ & $\mathbb{Z}$ \\
\hline$\left(0,3^{\prime}\right)$ & $\left(2,1^{\prime}\right)$ & $\left(0^{\prime}, 1\right)$ & $\left(2^{\prime}, 3\right)$ & $\mathbb{Z}$ & 0 & $\mathbb{Z}^{2}$ & $\mathbb{Z}$ \\
\hline$\left(0,1^{\prime}\right)$ & $\left(2,3^{\prime}\right)$ & $\left(0^{\prime}, 1\right)$ & $\left(2^{\prime}, 3\right)$ & $\mathbb{Z}$ & $\mathbb{Z} / 2$ & 0 & $\mathbb{Z}$ \\
\hline$\left(0,1^{\prime}\right)$ & $(2,1)$ & $\left(0^{\prime}, 3^{\prime}\right)$ & $\left(2^{\prime}, 3\right)$ & $\mathbb{Z}$ & $\mathbb{Z} / 2$ & 0 & $\mathbb{Z}$ \\
\hline
\end{tabular}

is commutative for all $k \in \mathbb{N}$ in view of the naturality of the cap-product. The right vertical arrow is an isomorphism by Poincaré duality. Hence, $H_{k}(f ; \mathbb{Z})$ : $H_{k}\left(N_{\pi} ; \mathbb{Z}\right) \longrightarrow H_{k}(M ; \mathbb{Z})$ is split surjective.

Moreover, covering theory shows that $\pi_{1}(f): \pi_{1}\left(N_{\pi}\right) \longrightarrow \pi_{1}(M)$ is surjective. Because $\pi_{1}\left(N_{\pi}\right)$ is cyclic, also $\pi_{1}(M)$ is cyclic and thus isomorphic to $H_{1}(M ; \mathbb{Z})$. As we have seen above, $H_{1}(f ; \mathbb{Z})$ is split surjective, and so $\pi_{1}(f): \pi_{1}\left(N_{\pi} ; \mathbb{Z}\right) \longrightarrow \pi_{1}(M)$ is split surjective. Therefore, the fundamental group of $M$ is isomorphic to

$$
0, \quad \mathbb{Z} / 2, \quad \mathbb{Z} / 3, \quad \text { or } \mathbb{Z} \text {. }
$$

Now the classification of oriented closed connected 3-manifolds [1] shows that $M$ is homotopy equivalent (even homeomorphic) to $S^{3}, \mathbb{R} P^{3}, L(3,1)$, or $S^{1} \times S^{2}$. As we know that $\left\|S^{3}\right\|_{\mathbb{Z}}=1 \neq 2$ the only remaining candidates for $M$ are $\mathbb{R} P^{3}, L(3,1)$, or $S^{1} \times S^{2}$, as claimed.

\subsection{Higher dimensions}

The example of the torus generalises as follows: 
Table 2. Integral homology of model spaces for cycles consisting of two singular 3-simplices with opposite signs.

\begin{tabular}{llllllll}
\hline \multicolumn{1}{l}{ Matching } & & & \multicolumn{3}{c}{$H_{*}(\cdot ; \mathbb{Z})$ in degree } \\
& & & & 0 & 1 & 2 & 3 \\
\hline$(0,1)$ & $(2,3)$ & $\left(1^{\prime}, 0^{\prime}\right)$ & $\left(3^{\prime}, 2^{\prime}\right)$ & $\mathbb{Z}^{2}$ & 0 & 0 & $\mathbb{Z}^{2}$ \\
$(0,3)$ & $(2,1)$ & $\left(1^{\prime}, 0^{\prime}\right)$ & $\left(3^{\prime}, 2^{\prime}\right)$ & $\mathbb{Z}^{2}$ & 0 & 0 & $\mathbb{Z}^{2}$ \\
$\left(0,0^{\prime}\right)$ & $(2,3)$ & $\left(1^{\prime}, 1\right)$ & $\left(3^{\prime}, 2^{\prime}\right)$ & $\mathbb{Z}$ & 0 & 0 & $\mathbb{Z}$ \\
$(0,3)$ & $\left(2,0^{\prime}\right)$ & $\left(1^{\prime}, 1\right)$ & $\left(3^{\prime}, 2^{\prime}\right)$ & $\mathbb{Z}$ & 0 & $\mathbb{Z}$ & $\mathbb{Z}$ \\
$\left(0,0^{\prime}\right)$ & $(2,1)$ & $\left(1^{\prime}, 3\right)$ & $\left(3^{\prime}, 2^{\prime}\right)$ & $\mathbb{Z}$ & 0 & 0 & $\mathbb{Z}$ \\
$(0,1)$ & $\left(2,0^{\prime}\right)$ & $\left(1^{\prime}, 3\right)$ & $\left(3^{\prime}, 2^{\prime}\right)$ & $\mathbb{Z}$ & 0 & 0 & $\mathbb{Z}$ \\
$\left(0,2^{\prime}\right)$ & $\left(2,0^{\prime}\right)$ & $\left(1^{\prime}, 3\right)$ & $\left(3^{\prime}, 1\right)$ & $\mathbb{Z}$ & 0 & $\mathbb{Z}$ & $\mathbb{Z}$ \\
$\left(0,0^{\prime}\right)$ & $\left(2,2^{\prime}\right)$ & $\left(1^{\prime}, 3\right)$ & $\left(3^{\prime}, 1\right)$ & $\mathbb{Z}$ & 0 & $\mathbb{Z}$ & $\mathbb{Z}$ \\
$\left(0,0^{\prime}\right)$ & $(2,3)$ & $\left(1^{\prime}, 2^{\prime}\right)$ & $\left(3^{\prime}, 1\right)$ & $\mathbb{Z}$ & 0 & 0 & $\mathbb{Z}$ \\
$\left(0,2^{\prime}\right)$ & $(2,3)$ & $\left(1^{\prime}, 0^{\prime}\right)$ & $\left(3^{\prime}, 1\right)$ & $\mathbb{Z}$ & 0 & 0 & $\mathbb{Z}$ \\
$(0,3)$ & $\left(2,2^{\prime}\right)$ & $\left(1^{\prime}, 0^{\prime}\right)$ & $\left(3^{\prime}, 1\right)$ & $\mathbb{Z}$ & 0 & $\mathbb{Z}$ & $\mathbb{Z}$ \\
$(0,3)$ & $\left(2,0^{\prime}\right)$ & $\left(1^{\prime}, 2^{\prime}\right)$ & $\left(3^{\prime}, 1\right)$ & $\mathbb{Z}$ & 0 & 0 & $\mathbb{Z}$ \\
$\left(0,2^{\prime}\right)$ & $(2,1)$ & $\left(1^{\prime}, 3\right)$ & $\left(3^{\prime}, 0^{\prime}\right)$ & $\mathbb{Z}$ & 0 & 0 & $\mathbb{Z}$ \\
$(0,1)$ & $\left(2,2^{\prime}\right)$ & $\left(1^{\prime}, 3\right)$ & $\left(3^{\prime}, 0^{\prime}\right)$ & $\mathbb{Z}$ & 0 & $\mathbb{Z}$ & $\mathbb{Z}$ \\
$(0,1)$ & $(2,3)$ & $\left(1^{\prime}, 2^{\prime}\right)$ & $\left(3^{\prime}, 0^{\prime}\right)$ & $\mathbb{Z}$ & 0 & 0 & $\mathbb{Z} 2$ \\
$\left(0,2^{\prime}\right)$ & $(2,3)$ & $\left(1^{\prime}, 1\right)$ & $\left(3^{\prime}, 0^{\prime}\right)$ & $\mathbb{Z}$ & 0 & $\mathbb{Z}$ & $\mathbb{Z}$ \\
$(0,3)$ & $\left(2,2^{\prime}\right)$ & $\left(1^{\prime}, 1\right)$ & $\left(3^{\prime}, 0^{\prime}\right)$ & $\mathbb{Z}$ & $\mathbb{Z}$ & $\mathbb{Z}$ & $\mathbb{Z}$ \\
$(0,3)$ & $(2,1)$ & $\left(1^{\prime}, 2^{\prime}\right)$ & $\left(3^{\prime}, 0^{\prime}\right)$ & $\mathbb{Z}$ & 0 & 0 & $\mathbb{Z}$ \\
$\left(0,2^{\prime}\right)$ & $(2,1)$ & $\left(1^{\prime}, 0^{\prime}\right)$ & $\left(3^{\prime}, 3\right)$ & $\mathbb{Z}$ & 0 & 0 & $\mathbb{Z}$ \\
$(0,1)$ & $\left(2,2^{\prime}\right)$ & $\left(1^{\prime}, 0^{\prime}\right)$ & $\left(3^{\prime}, 3\right)$ & $\mathbb{Z}$ & 0 & 0 & $\mathbb{Z}$ \\
$(0,1)$ & $\left(2,0^{\prime}\right)$ & $\left(1^{\prime}, 2^{\prime}\right)$ & $\left(3^{\prime}, 3\right)$ & $\mathbb{Z}$ & 0 & 0 & $\mathbb{Z}$ \\
$\left(0,2^{\prime}\right)$ & $\left(2,0^{\prime}\right)$ & $\left(1^{\prime}, 1\right)$ & $\left(3^{\prime}, 3\right)$ & $\mathbb{Z}$ & 0 & $\mathbb{Z}$ & $\mathbb{Z}$ \\
$\left(0,0^{\prime}\right)$ & $\left(2,2^{\prime}\right)$ & $\left(1^{\prime}, 1\right)$ & $\left(3^{\prime}, 3\right)$ & $\mathbb{Z}$ & 0 & 0 & $\mathbb{Z}$ \\
$\left(0,0^{\prime}\right)$ & $(2,1)$ & $\left(1^{\prime}, 2^{\prime}\right)$ & $\left(3^{\prime}, 3\right)$ & $\mathbb{Z}$ & 0 & 0 & $\mathbb{Z}$ \\
\hline
\end{tabular}

Proposition 4.6. For all $n \in \mathbb{N}_{>1}$ we have

$$
\left\|S^{1} \times S^{n-1}\right\|_{\mathbb{Z}}=2= \begin{cases}\left\|S^{1}\right\|_{\mathbb{Z}} \cdot\left\|S^{n-1}\right\|_{\mathbb{Z}} & \text { if } n \text { is odd } \\ 2 \cdot\left\|S^{1}\right\|_{\mathbb{Z}} \cdot\left\|S^{n-1}\right\|_{\mathbb{Z}} & \text { if } n \text { is even. }\end{cases}
$$

Proof. Theorem 1.1 shows that $\left\|S^{1} \times S^{n-1}\right\|_{\mathbb{Z}}>1$ because $S^{1} \times S^{n-1} \nsucceq S^{n}$. Therefore, it suffices to construct a fundamental cycle $\sigma-\sigma^{\prime}$ of $S^{1} \times S^{n-1}$ consisting of two singular simplices $\sigma, \sigma^{\prime}: \Delta^{n} \longrightarrow S^{1} \times S^{n-1}$. We now describe the construction of such singular simplices; to this end we construct the two factors of these maps separately.

The $S^{1}$-factors are defined on $\Delta^{n} \subset \mathbb{R}^{n+1}$ by

$$
\begin{aligned}
\sigma_{1}=\sigma_{1}^{\prime}: \Delta^{n} & \longrightarrow S^{1} \subset \mathbb{C} \\
\left(t_{0}, \ldots, t_{n}\right) & \longmapsto e^{2 \pi i \cdot \sum_{j=0}^{n} j \cdot t_{j}} .
\end{aligned}
$$

In order to understand the definition of the $S^{n-1}$-factors we first give the corresponding matchings: 
- If $n$ is even, then we consider the matching $\pi$ given by

$$
\begin{gathered}
0 \longleftrightarrow n^{\prime} \\
1 \longleftrightarrow 1^{\prime} \\
2 \longleftrightarrow 2^{\prime} \\
\vdots \\
n-1 \longleftrightarrow(n-1)^{\prime} \\
n \longleftrightarrow 0^{\prime} .
\end{gathered}
$$

Moreover, we define

$$
\begin{array}{lll} 
& d_{0}:=1=: d_{n}^{\prime} & d_{1}:=1=: d_{1}^{\prime} \\
\forall_{j \in\{2, \ldots, n-1\}} & d_{j}:=0=: d_{j}^{\prime} & d_{n}:=0=: d_{0}^{\prime} .
\end{array}
$$

- If $n$ is odd, then we consider the matching $\pi$ given by

$$
\begin{aligned}
0 \longleftrightarrow n \\
1 \longleftrightarrow 1^{\prime} \\
2 \longleftrightarrow 2^{\prime} \\
\vdots \\
n-1 \longleftrightarrow(n-1)^{\prime} \\
0^{\prime} \longleftrightarrow n^{\prime} .
\end{aligned}
$$

Moreover, we define

$$
\begin{array}{lll} 
& d_{0}:=1=: d_{n} & d_{1}:=0=: d_{1}^{\prime} \\
\forall_{j \in\{2, \ldots, n-1\}} & d_{j}:=0=: d_{j}^{\prime} & d_{0}^{\prime}:=0=: d_{n}^{\prime} .
\end{array}
$$

We choose $x_{0} \in S^{n-1}$ and a homeomorphism $\Delta^{n-1} / \partial \Delta^{n-1}$ that maps the point associated with $\partial \Delta^{n-1}$ to $x_{0}$. We denote the constant map with value $x_{0}$ by $c: \Delta^{n-1} \longrightarrow S^{n-1}$ and the projection corresponding to the above homeomorphism by $p: \Delta^{n-1} \longrightarrow \Delta^{n-1} / \partial \Delta^{n-1} \cong$ Top $S^{n-1}$. For $j \in\{0, \ldots, n\}$ we then define

$$
\sigma_{2, j}:= \begin{cases}c & \text { if } d_{j}=0 \\ p & \text { if } d_{j}=1\end{cases}
$$

Because $\sum_{j=0}^{n}(-1)^{j} \cdot d_{j}=0$, these maps can be extended to a continuous map $\sigma_{2}: \Delta^{n} \longrightarrow S^{n-1}$ with $\sigma_{2} \circ i_{j}=\sigma_{2, j}$ for all $j \in\{0, \ldots, n\}$. In the same way, we construct $\sigma_{2}^{\prime}: \Delta^{n} \longrightarrow S^{n-1}$ using $d_{0}^{\prime}, \ldots, d_{n}^{\prime}$.

Finally, we set

$$
\sigma:=\left(\sigma_{1}, \sigma_{2}\right) \quad \text { and } \quad \sigma^{\prime}:=\left(\sigma_{1}^{\prime}, \sigma_{2}^{\prime}\right) \text {. }
$$


Because the $d_{0}, \ldots, d_{n}$ and $d_{0}^{\prime}, \ldots, d_{n}^{\prime}$ are compatible with the matching $\pi$, the faces of $\sigma_{2}$ and $\sigma_{2}^{\prime}$ cancel according to $\pi$. Also $\sigma_{1}$ and $\sigma_{1}^{\prime}$ are compatible with the matching $\pi$ because for all $\left(t_{0}, \ldots, t_{n}\right) \in \Delta^{n}$ we have $\sum_{j=0}^{n} t_{j}=1$, and so $\sigma_{1} \circ i_{0}=\sigma_{1} \circ i_{n}$ etc. Thus, $\sigma-\sigma^{\prime}$ is a singular cycle on $S^{1} \times S^{n-1}$.

It remains to show that $\sigma-\sigma^{\prime}$ represents $\left[S^{1} \times S^{n-1}\right]_{\mathbb{Z}}$. To this end we choose singular cocycles $f_{1} \in C^{1}\left(S^{1} ; \mathbb{Z}\right)$ and $f_{2} \in C^{n-1}\left(S^{n-1} ; \mathbb{Z}\right)$ dual to $\left[S^{1}\right]_{\mathbb{Z}}$ and $\left[S^{n-1}\right]_{\mathbb{Z}}$ respectively; without loss of generality, we may assume that $f_{2}(c)=0$. Then the cohomological cross-product $f_{1} \times f_{2}$ is a cocycle on $S^{1} \times S^{n-1}$ that is dual to $\left[S^{1}\right]_{\mathbb{Z}} \times$ $\left[S^{n-1}\right]_{\mathbb{Z}}=\left[S^{1} \times S^{n-1}\right]_{\mathbb{Z}}$. Therefore, it suffices to show that $\left(f_{1} \times f_{2}\right)\left(\sigma-\sigma^{\prime}\right)=1$. Using the explicit description of the cohomological cross-product via the AlexanderWhitney map, we obtain

$$
\begin{aligned}
\left(f_{1} \times f_{2}\right)\left(\sigma-\sigma^{\prime}\right) & =(-1)^{n-1} \cdot\left(f_{1}\left(\sigma_{1}\right\rfloor_{1}\right) \cdot f_{2}\left({ }_{n-1}\left\lfloor\sigma_{2}\right)-f_{1}\left(\sigma_{1}^{\prime}\right\rfloor_{1}\right) \cdot f_{2}\left({ }_{n-1}\left\lfloor\sigma_{2}^{\prime}\right)\right) \\
& =(-1)^{n-1} \cdot\left(1 \cdot d_{n}-1 \cdot d_{n}^{\prime}\right) \\
& =1 .
\end{aligned}
$$

Therefore, $\sigma-\sigma^{\prime}$ is a fundamental cycle of $S^{1} \times S^{n-1}$.

In particular, in even dimensions spheres are not the only oriented closed connected manifolds with integral simplicial volume equal to 2 .

We now turn our attention to products of higher-dimensional spheres. In certain cases, we can show that these have integral simplicial volume larger than 2 . However, the general case is still wide open.

Lemma 4.7. Let $M$ be an oriented closed connected $n$-manifold with $\|M\|_{\mathbb{Z}}=2$. Then for all $k \in \mathbb{N}$ we have:

1. If $k$ is even, then $H_{k}(M ; \mathbb{Z})$ is cyclic.

2. If $k$ is odd, then $H_{k}(M ; \mathbb{Z})$ can be generated by two elements.

Proof. Because of $\|M\|_{\mathbb{Z}}=2$, there exist singular simplices $\sigma, \tau: \Delta^{n} \longrightarrow M$ such that $\sigma+\tau$ or $\sigma-\tau$ is a cycle in $C_{n}(M ; \mathbb{Z})$ representing $\pm[M]_{\mathbb{Z}}$. Without loss of generality, we may assume that $[\sigma+\varepsilon \cdot \tau]=[M]_{\mathbb{Z}} \in H_{n}(M ; \mathbb{Z})$ where $\varepsilon \in\{-1,1\}$.

It suffices to treat the case $k \in\{1, \ldots, n-1\}$. As in the proof of Lemma 3.1, we consider the Poincaré duality isomorphism

$$
\begin{aligned}
& \varphi_{k}:=\cdot \cap[M]_{\mathbb{Z}}: H^{n-k}(M ; \mathbb{Z}) \longrightarrow H_{k}(M ; \mathbb{Z}) \\
& {[f] \longmapsto(-1)^{(n-k) \cdot k} \cdot\left[\begin{array}{rl}
{\left[{ }_{n-k}\lfloor\sigma) \cdot \sigma\right\rfloor_{k}} \\
\left.+\varepsilon \cdot f\left({ }_{n-k}\lfloor\tau) \cdot \tau\right\rfloor_{k}\right]
\end{array}\right.}
\end{aligned}
$$

Clearly, the image of $\varphi_{k}$ can be generated by two elements or less. If $k$ is even, then neither $\sigma\rfloor_{k}$ nor $\left.\tau\right\rfloor_{k}$ is a cycle (Lemma 2.3); hence, there is (up to integral 
rescaling) at most one $\mathbb{Z}$-linear combination of $\sigma\rfloor_{k}$ and $\left.\tau\right\rfloor_{k}$ that is a cycle. Thus, the image of $\varphi_{k}$ is cyclic. Because $\varphi_{k}$ is an isomorphism and hence surjective, the result follows.

In general, these bounds cannot be improved as the example of the torus in dimension 2 shows (Proposition 4.3).

Proposition 4.8. Let $n \in \mathbb{N}$ be even. Then

$$
\left\|S^{n} \times S^{n}\right\|_{\mathbb{Z}} \geq 4=\left\|S^{n}\right\|_{\mathbb{Z}} \cdot\left\|S^{n}\right\|_{\mathbb{Z}}
$$

Proof. On the one hand, $\left\|S^{n} \times S^{n}\right\|_{\mathbb{Z}}$ is even (Lemma 4.2). On the other hand, by Lemma 4.7 , we have $\left\|S^{n} \times S^{n}\right\|_{\mathbb{Z}}>2$ because $H_{n}\left(S^{n} \times S^{n} ; \mathbb{Z}\right) \cong \mathbb{Z}^{2}$ and $n$ is assumed to be even.

Remark 4.9. We have

$$
\left\|S^{2} \times S^{4}\right\|_{\mathbb{Z}} \geq 4=\left\|S^{2}\right\|_{\mathbb{Z}} \cdot\left\|S^{4}\right\|_{\mathbb{Z}}
$$

Indeed, $\left\|S^{2} \times S^{4}\right\|_{\mathbb{Z}}$ is even by Lemma 4.2 . Because this integral simplicial volume is non-zero it suffices to prove that $\left\|S^{2} \times S^{4}\right\|_{\mathbb{Z}} \neq 2$.

As in Remark 4.5 we consider model spaces for cycles consisting of two singular 6-simplices. All such cycles are modelled by matchings that are bijections

$$
\left\{0,2,4,6,1^{\prime}, 3^{\prime}, 5^{\prime}\right\} \longrightarrow\left\{1,3,5,0^{\prime}, 2^{\prime}, 4^{\prime}, 6^{\prime}\right\}
$$

Calculating the homology of the model spaces of all possible matchings (via a computer) shows that they all have second integral homology that does not surject onto $\mathbb{Z}$. On the other hand, if $\left\|S^{2} \times S^{4}\right\|_{\mathbb{Z}}$ were equal to 2 , then the cap-product argument of Remark 4.5 shows that the model map on second integral homology would need to be surjective. This shows that $\left\|S^{2} \times S^{4}\right\|_{\mathbb{Z}} \neq 2$.

Question 4.10. (integral simplicial volume of products) If $M$ and $N$ are oriented closed connected manifolds, then the ordinary simplicial volume (with $\mathbb{R}$-coefficients) satisfies [10][2]

$$
\|M\| \cdot\|N\| \leq\|M \times N\| \leq\left(\begin{array}{c}
\operatorname{dim} M+\operatorname{dim} N \\
\operatorname{dim} N
\end{array}\right) \cdot\|M\| \cdot\|N\| .
$$

The upper estimate is based on the homological cross-product and works in the same way also for the integral simplicial volume. But the lower estimate depends on the Hahn-Banach theorem, and the argument does not carry over to the integral setting. Hence, one might ask whether also $\|M\|_{\mathbb{Z}} \cdot\|N\|_{\mathbb{Z}} \leq\|M \times N\|_{\mathbb{Z}}$ holds. 


\section{Integral simplicial volume is not computable}

We will now briefly introduce the relevant setup for computability and derive the non-computability statement of Corollary 1.3 from Theorem 1.1:

For $n \in \mathbb{N}$ we let $K_{n}$ be the countable set of all finite $n$-dimensional simplicial complexes whose vertices form a subset of $\mathbb{N}$ and we let $T_{n}$ be the subset of all such complexes whose geometric realisation is an oriented closed connected topological $n$-manifold. We now consider an injection $i_{n}: K_{n} \longrightarrow \mathbb{N}$ with the property that for every $K \in K_{n}$ the number $i_{n}(K)$ can be calculated by integer arithmetic from the set of simplices, and conversely such that for every $m \in i_{n}\left(K_{n}\right)$ the complex $K$ with $i_{n}(K)=m$ can be reconstructed by integer arithmetic. For example, such a map $i_{n}$ can be obtained by considering suitable products of prime numbers.

Using $i_{n}$, we can interpret notions from computability on $T_{n}$ : A function $f: T_{n} \longrightarrow \mathbb{N}$ is computable [resp. semi-computable], if the associated function $f \circ$ $i_{n}^{-1}: i_{n}\left(T_{n}\right) \longrightarrow \mathbb{N}$ is a partial recursive [resp. $\mu$-recursive] function $\mathbb{N} \longrightarrow \mathbb{N}$ with domain $i_{n}\left(T_{n}\right)$. A subset $A \subset T_{n}$ is called decidable [resp. semi-decidable] if there is a computable [resp. semi-computable] function $f: T_{n} \longrightarrow \mathbb{N}$ satisfying $A=f^{-1}(0)$ and $T_{n} \backslash A=f^{-1}(1)$. For the exact definition of $(\mu-)$ recursive functions and the relation with Turing machines we refer to the literature [4].

We can now formulate Corollary 1.3 in a more precise way:

Corollary 5.1. (non-computability) Let $n \in \mathbb{N}_{\geq 5}$ be odd.

1. Then the set

$$
\left\{K \in T_{n}\left|\||K|\|_{\mathbb{Z}}=1\right\}\right.
$$

is not decidable.

2. In particular, the function

$$
\begin{aligned}
I_{n}: T_{n} & \longrightarrow \mathbb{N} \\
K & \longrightarrow\||K|\|_{\mathbb{Z}}
\end{aligned}
$$

is not computable.

Proof. Ad 1. In view of Theorem 1.1, we have

$$
\left\{K \in T_{n}\left|\||K|\|_{\mathbb{Z}}=1\right\}=\left\{K \in T_{n}|| K \mid \simeq S^{n}\right\},\right.
$$

which is known to be not decidable [20].

$A d$ 2. If $I_{n}$ were computable, then the set $I_{n}^{-1}(1) \subset T_{n}$ were decidable, which contradicts the first part. Hence, $I_{n}$ is not computable. 
Remark 5.2. (semi-computability) For all $n \in \mathbb{N}$ the function $I_{n}: T_{n} \longrightarrow \mathbb{N}$ is semi-computable in the following sense: If $m \in \mathbb{N}$, then the set

$$
I_{n}^{-1}(\{0, \ldots, m\})=\left\{K \in T_{n}\left|\||K|\|_{\mathbb{Z}} \leq m\right\}\right.
$$

is semi-decidable: Let $K \in T_{n}$. If $c \in C_{n}(|K| ; \mathbb{Z})$ is a cycle, then the simplicial approximation theorem and an inductive construction of (relative) homotopies shows that there is a $k \in \mathbb{N}$ and a singular chain $c^{\prime} \in C_{n}(|K| ; \mathbb{Z})$ with the following properties:

- We have $[c]=\left[c^{\prime}\right]$ in $H_{n}(|K| ; \mathbb{Z})$ and $\left|c^{\prime}\right|_{1} \leq|c|_{1}$.

- The chain $c^{\prime}$ is a generalised simplicial chain, i.e., it consists of singular simplices $\Delta^{n} \longrightarrow K$ that are geometric realisations of simplicial maps from the $k$-fold barycentric subdivision of $\Delta^{n}$ (viewed as simplicial complex in the standard way) to $K$.

Moreover, all the following operations can be performed by Turing machines: enumerate all barycentric subdivisions of $\Delta^{n}$, enumerate all simplicial maps between two finite simplicial complexes, check whether a generalised simplicial $\mathbb{Z}$-chain on a finite simplicial complex in $T_{n}$ is a fundamental cycle, and compute the $\ell^{1}$-norm of a generalised simplicial singular $\mathbb{Z}$-chain.

Hence, there is a Turing machine acting on inputs $K$ from $T_{n}$ such that: If $\||K|\|_{\mathbb{Z}} \leq m$, then the Turing machine stops and accepts $K$. If $\||K|\|_{\mathbb{Z}}>m$, then the Turing machine does not stop or, if it stops, does not accept $K$. In other words, the set $I_{n}^{-1}(\{0, \ldots, m\})$ is semi-decidable.

However, in certain restricted situations, integral simplicial volume at least is a finite-to-one map:

Proposition 5.3. Let $m \in \mathbb{N}$. Then there exist only finitely many homeomorphism types of oriented closed connected 3-manifolds $M$ with $\|M\|_{\mathbb{Z}} \leq m$.

The proof uses models of singular cycles, similar to the ones discussed in the previous sections. A more detailed description of such combinatorial models of cycles and their associated homology classes can be found in the discussion of weak flexibility [17, Section 5.1].

Proof. Let $n \in \mathbb{N}$. Then there exist only finitely many different combinatorial types of singular $n$-cycles with $\mathbb{Z}$-coefficients and $\ell^{1}$-norm at most $m$. These finitely many combinatorial types can be combined into a finite connected simplicial complex $X$ and a class $\alpha \in H_{n}(X ; \mathbb{Z})$ with the following property: If $M$ is an oriented closed connected $n$-manifold with $\|M\|_{\mathbb{Z}} \leq m$, then there is a continuous map $f: X \longrightarrow M$ satisfying

$$
H_{n}(f ; \mathbb{Z})(\alpha)=[M]_{\mathbb{Z}}
$$


Now let $n=3$; in particular, $n \leq 5$. Then by Thom's work [23] on representing homology classes through manifolds, there is an oriented closed connected $n$-manifold $N$ and a continuous map $g: N \longrightarrow X$ with

$$
H_{n}(g ; \mathbb{Z})\left([N]_{\mathbb{Z}}\right)=\alpha .
$$

Composing these maps, we see that $N$ dominates every oriented closed connected 3-manifold $M$ with $\|M\|_{\mathbb{Z}} \leq m$ through a map of degree 1 . However, every oriented closed connected 3-manifold can dominate only finitely many other homeomorphism types of oriented closed connected 3-manifolds [14][3].

Acknowledgements. I would like to thank the anonymous referees for their constructive suggestions and corrections.

\section{References}

1. Aschenbrenner, M., Friedl, S. and Wilton, H., 3-Manifold Groups, EMS Ser. Lect. Math., EMS, 2015.

2. Benedetti, R. and Petronio, C., Lectures on Hyperbolic Geometry, Universitext, Springer, 1992.

3. Boileau, M., Hyam Rubinstein, J. and Wang, S., Finiteness of 3-manifolds associated with non-zero degree mappings, Comment. Math. Helv. 89 (2014), $33-68$.

4. Boolos, G. S., Burgess, J. P. and Jeffrey, R. C., Computability and Logic, fifth ed., Cambridge University Press, 2007.

5. FAuser, D. and LÖH, C., Exotic finite functorial semi-norms on singular homology, to appear in Glasgow Mathematical Journal. arXiv: 1605.04093 [math.GT].

6. Félix, Y., Halperin, S. and Thomas, J.-C., Rational Homotopy Theory, Grad. Texts in Math. 205, Springer, 2001.

7. Francaviglia, S., Frigerio, R. and Martelli, B., Stable complexity and simplicial volume of manifolds, J. Topol. 5 (2012), 977-1010.

8. Frigerio, R., Löh, C., Pagliantini, C. and Sauer, R., Integral foliated simplicial volume of aspherical manifolds, Israel J. Math. 216 (2016), 707-751.

9. Gaboriau, D., Invariants $\ell^{2}$ de relations d'équivalence et de groupes, Inst. Hautes Études Sci. Publ. Math. 95 (2002), 93-150.

10. Gromov, M., Volume and bounded cohomology, Inst. Hautes Études Sci. Publ. Math. 56 (1983), 5-99.

11. Gromov, M., Asymptotic invariants of infinite groups, in Geometric Group Theory (Sussex, 1991), London Math. Soc. Lectures Notes Ser. 2, pp. 1-295, Cambridge University Press, 1993. 182.

12. Gromov, M., Metric Structures for Riemannian and Non-Riemannian Spaces with Appendices, Progr. Math. 152, Birkhäuser, 1999.

13. JungreIs, D., Chains that realize the Gromov invariant of hyperbolic manifolds, Ergodic Theory Dynam. Systems 17 (1997), 643-648. 
14. LiU, Y., Finiteness of nonzero degree maps between three-manifolds, Preprint, 2011. arXiv: 1107.5855 [math.GT].

15. LöH, C., Simplicial volume, Bull. Man. Atl. (2011), 7-18.

16. LÖH, C. and PAGLiantini, C., Integral foliated simplicial volume of hyperbolic 3-manifolds, Groups Geom. Dyn. 10 (2016), 825-865.

17. LöH, C., Finite functorial semi-norms and representability, Int. Math. Res. Not. 2016 (2016), 3616-3638.

18. LüCK, W., Approximating $L^{2}$-invariants by their finite-dimensional analogues, Geom. Funct. Anal. 4 (1994), 455-481.

19. LüCK, W., $L^{2}$-Invariants: Theory and Applications to Geometry and K-Theory, Ergeb. Math. Grenzgeb. (3), Springer, 2002.

20. Markov, A., The insolubility of the problem of homeomorphy, Dokl. Akad. Nauk SSSR 121 (1958), 218-220.

21. Munkholm, H. J., Simplices of maximal volume in hyperbolic space, Gromov's norm, and Gromov's proof of Mostow's rigidity theorem (following Thurston), in Topology Symposium (Siegen, 1979), Lecture Notes in Math. 788, pp. 109124, Springer, 1980.

22. Schmidt, M., $L^{2}$-Betti Numbers of $\mathcal{R}$-spaces and the Integral Foliated Simplicial Volume, Ph.D. Thesis, WWU Münster, 2005. Available online at http:// nbn-resolving. de/urn:nbn:de:hbz:6-05699458563.

23. Тном, R., Quelques propriétés globales des variétés différentiables, Comment. Math. Helv. 28 (1954), 17-86.

24. WeInBerger, S., Computers, rigidity, and moduli, The large-scale fractal geometry of Riemannian moduli space, Princeton University Press, 2005, M. B. Porter Lectures.

Clara Löh

Fakultät für Mathematik

Universität Regensburg

DE-93040 Regensburg

Germany

clara.loeh@mathematik.uni-regensburg.de

http://www.mathematik.uni-regensburg.de/loeh

Received March 15, 2017

in revised form July 31, 2017 